\title{
Synthesis of Antenna Systems According to the Desired Amplitude Radiation Characteristics
}

\author{
Mykhaylo Andriychuk
}

Additional information is available at the end of the chapter

http://dx.doi.org/10.5772/46164

\section{Introduction}

The problems of antenna synthesis, which use the amplitude RP as input information, are often used in the process of antenna design for many practical applications [4, 16, 27, 31]. In spite of the fact that the respective mathematical problems are ill-posed [32] and they have the branching solutions [29], the antenna synthesis according to the desired amplitude characteristics is very useful and perspective.

As a rule, the branching of solutions depends on the properties of prescribed amplitude $\mathrm{RP}$, geometrical and physical parameters of the considered antenna. The methods of nonlinear functional analysis [23] allowing to localize the branching solutions are applied for investigation of solutions and determination of their number and qualitative characteristics. Such approach too much simplifies determination of the optimal solutions by the numerical methods. The iterative processes for the numerical solving of the corresponding non-linear equations were elaborated in $[3,8,11]$.

The Chapter is organized as follows.

In Section 2 we derive the main formulas for RP of antennas and introduce the objective functionals for the synthesis problem. Also in Section 2 we consider the variational statement of problems and derive the fundamental nonlinear equations of the synthesis.

Section 3 contains the application of the proposed approach to several types of antennas. Depending on the restrictions which are imposed on the sought distribution of current or field in the antenna elements and type of antenna, the problems of amplitude-phase, amplitude, and phase synthesis are considered for the specific antennas. The methods of successive approximations are applied for solving the derived non-linear integral equations; the convergence of the elaborated methods is discussed. The direct optimization of the proposed functionals by the gradient methods is performed and successfully applied to solving the amplitude and phase synthesis problems.

In Section 4 conclusions are formulated. 


\section{The theoretical background of the synthesis problems}

In this Section, we present the necessary information about the properties of the electromagnetic (EM) field in far zone, introduce the variational approach to the antenna synthesis problems, as well as discuss the arising nonlinear integral and matrix equations.

\subsection{RP of electromagnetic field}

The EM field in the non-limited homogeneous medium satisfies the Maxwell equations [25]

$$
\begin{gathered}
\operatorname{rot} H=i k \varepsilon E+\frac{4 \pi}{c} I, \\
\operatorname{rot} E=-i k \mu H, \\
\operatorname{div} E=\rho / \varepsilon, \\
\operatorname{div} H=0,
\end{gathered}
$$

where $E$ is a component of electric field, $H$ is a component of magnetic field, $I$ is the extrinsic current density, $\rho$ is the volume density of electric charge.

The scalar and vector potentials are used for solving the equations (1)-(4). Introducing the vector potential $A^{e}$ as [34]

$$
H=\operatorname{rot} A^{e},
$$

we satisfy the equation (4). Substituting (5) into (2), we receive the equation

$$
\operatorname{rot}\left(E+i \omega \mu A^{e}\right)=0,
$$

which testifies that the vector field in the parenthesis of (6) is potential. This yields the equation

$$
E=-i \omega \mu A^{e}-\operatorname{grad} \varphi^{e},
$$

where $\varphi^{e}$ is the scalar potential. Substituting (5) and (7) into (1), we receive the following equation for $I$ :

$$
\nabla^{2} A^{e}+\omega^{2} \varepsilon \mu A^{e}-\operatorname{grad}\left(\operatorname{div} A^{e}+i \omega \varphi^{e}\right)=-I .
$$

Using Lorentz lemma [15]

$$
\operatorname{div} A^{e}+i \omega \varepsilon \varphi^{e}=0,
$$

we receive the inhomogeneous Helmholtz equation for the vector potential

$$
\nabla^{2} A^{e}+k^{2} A^{e}=-I
$$

where $k=\omega \sqrt{\varepsilon \mu}$ is a propagation coefficient. For a free space, the values $\varepsilon$ and $\mu$ are real and related with the light velocity as $c=1 / \sqrt{\varepsilon \mu}$, and coefficient $k=\omega / c=2 \pi / \lambda$ is the wavenumber, $\lambda$ is the length of wave.

The vector potential $A^{e}$ in the arbitrary observation point $Q(x, y, z)$ is determined by formula [26]

$$
A^{e}(x, y, z)=\frac{1}{4 \pi} \int_{V} I\left(x^{\prime}, y^{\prime}, z^{\prime}\right) \frac{-e^{i k R_{P Q}}}{R_{P Q}} d V,
$$


where $R_{P Q}=\sqrt{\left(x-x^{\prime}\right)^{2}+\left(y-y^{\prime}\right)^{2}+\left(z-z^{\prime}\right)^{2}}$ is the distance between the observation point $Q(x, y, z)$ and integration point $P\left(x^{\prime}, y^{\prime}, z^{\prime}\right), I\left(x^{\prime}, y^{\prime} z^{\prime}\right)$ is the density of current in the domain $V$.

Using formulas (5), (7), and (11), we receive the solution to system (1)-(4) in the term of electric vector potential

$$
\begin{gathered}
E=-i \omega \mu A^{e}-\frac{1}{\omega \varepsilon} \operatorname{graddiv} A^{e}, \\
H=\operatorname{rot} A^{e} .
\end{gathered}
$$

In the process of solving the synthesis problem, the representation of field in far zone is of interest. Using the approximate representation of distance $R_{P Q}$ in far zone, we receive the formula for vector potential in this region

$$
A_{\infty}^{e}(Q)=\frac{-e^{i k r}}{4 \pi r} \int_{V} I\left(x^{\prime}, y^{\prime}, z^{\prime}\right) e^{i k r^{\prime} \cos \alpha} d V+O\left(\frac{1}{r^{2}}\right),
$$

where $\alpha$ is the angle between the vectors, directed into observation and integration point, $r^{\prime}$ and $r$ are the radius-vector of the points $P$ and $Q$, respectively.

Substituting (14) into (13) and neglecting by terms of $O\left(\frac{1}{r^{2}}\right)$ order, we receive the formulas for components of magnetic field $H$ in term of vector potential $A_{\infty}^{e}$. These formulas in the spherical coordinates have form

$$
H_{r}=0, \quad H_{\theta}=i k A_{\infty \varphi}^{e}, \quad H_{\varphi}=-i k A_{\infty \theta}^{e} .
$$

Consequently, the formulas for $E$ are the following

$$
E_{r}=0, \quad E_{\theta}=-i \omega \mu A_{\infty \theta}^{e}, \quad E_{\varphi}=-i \omega \mu A_{\infty \varphi}^{e} .
$$

Using formulas (15), (16) and relation

$$
E=-\frac{1}{\omega \varepsilon} \operatorname{rot} H
$$

we receive the formulas for non-zero $E$ components of EM field

$$
\begin{aligned}
& E_{\theta}(r, \theta, \varphi)=-i \omega \mu \frac{e^{-i k r}}{4 \pi r} f_{\theta}(\theta, \varphi), \\
& E_{\varphi}(r, \theta, \varphi)=-i \omega \mu \frac{e^{-i k r}}{4 \pi r} f_{\varphi}(\theta, \varphi) .
\end{aligned}
$$

Function $\frac{e^{-i k r}}{4 \pi r}$ is a spherical wave and it depends on $r$ only. The second terms in (18) and (19) are the functions of angular coordinates of the observation point and are determined by the current $I\left(x^{\prime}, y^{\prime}, z^{\prime}\right)$

$$
\begin{aligned}
& f_{\theta}(\theta, \varphi)=\int_{V}\left[I_{x}\left(x^{\prime}, y^{\prime}, z^{\prime}\right) \cos \theta \cos \varphi+I_{y}\left(x^{\prime}, y^{\prime}, z^{\prime}\right) \cos \theta \sin \varphi-\right. \\
& \left.I_{z}\left(x^{\prime}, y^{\prime}, z^{\prime}\right) \sin \theta\right] e^{i k\left(x^{\prime} \sin \theta \cos \varphi+y^{\prime} \sin \theta \sin \varphi+z^{\prime} \cos \theta\right)} d V, \\
& f_{\varphi}(\theta, \varphi)=\int_{V}\left[I_{y}\left(x^{\prime}, y^{\prime}, z^{\prime}\right) \cos \varphi-I_{x}\left(x^{\prime}, y^{\prime}, z^{\prime}\right) \sin \varphi\right] \times \\
& e^{i k\left(x^{\prime} \sin \theta \cos \varphi+y^{\prime} \sin \theta \sin \varphi+z^{\prime} \cos \theta\right)} d V \text {. }
\end{aligned}
$$


The functions (20) and (21) characterize the angular distribution of $E$ components in far zone. In such a way, the vectors $E$ and $H$ in far zone are expressed by formulas [4]

$$
\begin{gathered}
E(r, \theta, \varphi)=-i \omega \mu \frac{e^{-i k r}}{4 \pi r}\left\{0, f_{\theta}(\theta, \varphi), f_{\varphi}(\theta, \varphi)\right\}, \\
H(r, \theta, \varphi)=i k \frac{e^{-i k r}}{4 \pi r}\left\{0, f_{\varphi}(\theta, \varphi),-f_{\theta}(\theta, \varphi)\right\} .
\end{gathered}
$$

The functions $f_{\theta}(\theta, \varphi)$ and $f_{\varphi}(\theta, \varphi)$ are defined as the RPs. Function

$$
N(\theta, \varphi)=\left|f_{\theta}(\theta, \varphi)\right|^{2}+\left|f_{\theta}(\theta, \varphi)\right|^{2}
$$

is the power RP and it characterizes the angular distribution of power intensity radiation. The functions $f_{\theta}(\theta, \varphi), f_{\varphi}(\theta, \varphi)$, and $N(\theta, \varphi)$ are used in the process of formulating and solving the synthesis problems for various antennas.

\subsection{Variational statement of the synthesis problems}

Abstracting of the specific type of antenna, we present the functions $f_{\theta}$ and $f_{\varphi}$ in the formulas (22)-(24) by the linear operator $\mathbf{A}=\left\{A_{\theta}, A_{\varphi}\right\}$ :

$$
\mathbf{f}=\mathbf{A} I, \quad\left(f_{v}=A_{v} I, \quad v=\theta, \varphi\right),
$$

acting from some complex Hilbertian space $H_{I}$, to which the distribution functions of current or field belong, into the complex space $C_{f}^{2}=C[\Omega] \oplus C[\Omega]$ of vector-valued continuous functions on the compact $\bar{\Omega} \in \mathrm{R}^{2}$ (or $\bar{\Omega} \in \mathrm{R}^{1}$ ) [29]. The form and properties of the operators $A_{v}$ depend on a type and geometry of antenna. In many practical applications, one can reduce the synthesis problem to separate consideration of the $f_{\theta}$ and $f_{\varphi}$ components. This allows to reduce the synthesis problem to the scalar one. In that way, we will use more simple formula

$$
f=A I
$$

for operator expression of the RP $f$.

The specific form of the operator $A$ depends on the antenna type. This operator is integral for the continuous antennas. As an example, for a cylindrical antenna with curvilinear generatrix and with current polarized along the cylinder axis, the RP in the transversal plane has form [21]

$$
f=A I:=\int_{S} I(S) e^{i k r\left(\varphi^{\prime}\right) \cos \left(\varphi-\varphi^{\prime}\right)} d S_{\varphi^{\prime}},
$$

where $f$ and $I$ are nonzero components $f_{\theta}$ and $I_{z}$ respectively; $\varphi$ is the angular coordinate of the point in far zone, $\varphi^{\prime}$ is the angular coordinate of the point in antenna, $r=r\left(\varphi^{\prime}\right)$ describes the generatrix in polar coordinates, $d S_{\varphi^{\prime}}=\sqrt{r^{2}+\left(d r / d \varphi^{\prime}\right)^{2}}$ is an element of arc. In the case of array, the operator $A$ is described by a finite sum.

In the previous subsection, we consider the direct external problem of electrodynamics consisting of determination of the asymptotic (RP) of EM field in far zone. The inverse problem, namely determination of such a current $I$ that create EM field with the desired RP $f$, is of specific interest in the antenna design. The characteristic parameters of antenna can be 
fixed or be subject to determination in process of solving this problem. In such interpretation, the inverse problem is defined as the synthesis problem, namely the problem of determination of the current according to the desired RP.

The RPs are due to satisfy a series of requirements for the main lobe and sidelobes. In one case, the RP with narrow main beam is required, another time, this beam should have the specific wide (for example, cosecant) form; the sidelobes be as low as possible.

The angular distribution of the radiation power is characterized by the amplitude of RP, but not the whole RP. Therefore, only amplitude $|f|$ of function $f$ is interesting in the process of statement and solving the synthesis problem. In this case, the freedom of choice of the phase $\arg f$ of function $f$ is used for better approximation to the amplitude RP. In the synthesis theory, function $|f|$ is amplitude RP, and function $\arg f$ is phase RP.

In this way, in the process of synthesis we prescribe not the whole complex function $f$, but only its amplitude. We denote this function as $F$, the created (synthesized) by antenna amplitude $\mathrm{RP}$ is denoted by $|f|$. The both functions are real and positive. Of course, these functions can not coincide in any real-world situation. This fact yields to use the variational statement of the synthesis problem. In such statement, one requires not the whole coincidence of the functions $|f|$ and $F$, but the better approximation of function $|f|$ to $F$ in a certain sense only. The mean-square deviation of both RPs is used as the criterion of optimization.

Let us introduce the Hilbertian spaces of radiation patterns $H_{f}$ and currents $H_{I}$. Let $(\cdot, \cdot)_{f}$ be an inner product in $H_{f}$, and norm of $f$ is defined as [4]

$$
\|f\|^{2}=(f, f)_{f}
$$

The functional

$$
\sigma=(F-s|f|, F-s|f|)_{f}
$$

is used in most cases as optimization criterion. Dependence of $\sigma$ on the sought distribution of the current $I$ in antenna is determined by formula (26). The additional multiplier $s$ in (29) can be either prescribed (for example, $s=1$ ) or determined from the condition $\partial \sigma / \partial s=0$. In the latter case

$$
s=(F,|f|)_{f} /(f, f)_{f} .
$$

Let us introduce the normalization $(F, F)_{f}=1$, then (29) can be written as

$$
\sigma=1-Q \kappa^{2}
$$

where

$$
\begin{gathered}
Q=\left\|I||^{2} /\right\| f \|^{2}, \\
\kappa=(F,|f|)_{f} /\|I\|,
\end{gathered}
$$

and $\|I\|$ is the norm of current, determined by inner product $(\cdot, \cdot)_{I}$ in the Hilbertian space $H_{I}$ of currents: $\|I\|^{2}=(I, I)_{I}$. The factor $Q$ characterizes the goodness (reactivity) of antenna, and $\kappa$ determines its power efficiency, namely the part of power radiated by the RP.

The generalized functional, allowing to diminish the mean-square deviation of RPs and relative norm of current, has the form

$$
\sigma_{t}=(F-|f|, F-|f|)_{f}+t|| I||^{2} .
$$


Choosing the weight multiplier $t$, one can regularize the value of mean-square deviation of the RPs and norm of current. Similar functional appears when the method of Lagrange multipliers [16] is used to solving the conditional extremum problems. Simultaneously, one can use some weight function $p \geq 0$ in the definition (28) and to improve the approximation to prescribed amplitude RP $F$ in the appointed angular range.

\subsection{The fundamental equations of synthesis}

Let us demonstrate how one can receive the respective Lagrange-Euler's equation [17] in the process of minimization of functional $\sigma$. Let $s=1$. It is known [30] that the equality to zero of functional's gradient is requirement of its extremum. This yields determination of the maximum of the following value

$$
\frac{\partial \sigma}{\partial z}=\frac{1}{\|z\|} \lim _{\varepsilon \rightarrow 0} \frac{\sigma(I+\varepsilon z)-\sigma(I)}{\varepsilon} .
$$

In order to determine the derivative $\frac{\partial \sigma}{\partial z}$, one requires to factorize $\sigma(I+\varepsilon z)$ in series relatively to $\varepsilon$

$$
\sigma(I+\varepsilon z)=\sigma(I)+\varepsilon \delta \sigma(I, z)+O\left(\varepsilon^{2}\right)
$$

and to extract the linear term $\delta \sigma$. Evidently, $\frac{\partial \sigma}{\partial z}=\frac{\delta \sigma}{\|z\|}$. The increment of amplitude RP $|f|$ should be known in order to calculate $\delta \sigma$. This increment has the form [4]

$$
|A(I+\varepsilon z)|=|f|+\varepsilon \operatorname{Re}\left[A(z) e^{-i \arg f}\right]+O\left(\varepsilon^{2}\right) .
$$

Substituting this expression into (35), we receive

$$
\frac{\partial \sigma}{\partial z}=-2\left(F, \operatorname{Re}\left[A(z) e^{-i \arg f}\right]\right)_{f}+2 \operatorname{Re}(f, A z)_{f},
$$

or

$$
\frac{\partial \sigma}{\partial z}=-2 \operatorname{Re}\left[\left(A^{*}\left(f-F e^{-i \arg f}\right), z\right)_{I}\right]
$$

where $A^{*}$ is an operator adjoint to $A$ in the following sense:

$$
\left(A I_{1}, f_{2}\right)_{f}=\left(I_{1}, A^{*} f_{2}\right)_{I} .
$$

If $A$ is an integral operator then $A^{*}$ is the same with the complex conjugate kernel and integration with respect to the second argument.

Using the Cauchy-Bunyakovsky-Schwarz inequality [10] and maximizing $\frac{\partial \sigma}{\partial z}$, we receive the expression for gradient of $\sigma$ :

$$
z=A^{*}\left(f-F e^{-i \arg f}\right),
$$

which is used usually for the numerical minimization of $\sigma$. In order to receive the Lagrange-Euler's equation one should equate to zero the function $z$ (condition of $\sigma$ minimum)

$$
A^{*}\left(f-F e^{-i \arg f}\right)=0 .
$$

The equation (42) contains $f$ as an unknown function. One can turn out that its solutions represent the unreliazable patterns [20]. In order to avoid such solutions, it is necessary to 
substitute formula (26) into (42) instead of $f$. In this way, we receive the integral equation for the optimal current distribution

$$
A^{*} A I=A^{*} A\left(F e^{-i \arg A I}\right) .
$$

If operator $A$ is integral (consequently, $A^{*}$ is also integral) then (43) is the nonlinear Hammerstein equation [38].

One can receive the similar expression for $z$ in the process of $\kappa$ maximization:

$$
z=A^{*}\left(F e^{-i \arg f}\right)-I \text {. }
$$

Equating the right part of (44) to zero, we receive the explicit expression for $I$ :

$$
I=A^{*}\left(F e^{-i \arg f}\right) .
$$

Equations (26) and (45) yield the system of nonlinear integral equations for the optimal current $I$ and RP $f$ created by it.

System of nonlinear equations (26), (45) can be reduced to one nonlinear equation

$$
f=A A^{*}\left(F e^{-i \arg f}\right) .
$$

Since right hand side of (46) is a result of acting by the operator $A$ on some function, any solution to this equation represents the realizable RP. Once this equation is solved, the optimal distribution of current is determined by formula (45). The equation (46) is more simple than system (26), (45) because determination of its solution does not require additional operation of $A$ and $A^{*}$.

Taking into account the above considerations, we receive the following nonlinear Hammerstein equation of the second kind for the RP $f$

$$
t f+A A^{*} f=A A^{*}\left(F e^{-i \arg f}\right)
$$

in the case of functional $\sigma_{t}$ minimization. As in the case of (46), the solution to this equation (at $t \neq 0$ ) represents the realizable RP. Having the solution to (47), we determine the optimal current distribution by formula

$$
I=-1 / t A^{*}\left(f-F e^{-i \arg f}\right) .
$$

The integral equations (43), (46), and (47) are the fundamental equations for the synthesis problems according to the prescribed amplitude RP F.

\subsection{The numerical solution of the integral equations}

The equations (43), (46), and (47) are solved numerically by the method of successive approximations. The new approximation is determined by explicit formula

$$
f_{n+1}=A A^{*}\left(F e^{-i \arg f_{n}}\right)
$$

in the process of $\kappa$ maximization. The equation

$$
A^{*} A I_{n+1}=A^{*} A\left(F e^{-i \arg A I_{n}}\right)
$$


is solved when the synthesis problem is formulated in the term of functional $\sigma$ minimization. If functional $\sigma_{t}$ is minimized then the respective iterative procedure has form

$$
t f_{n+1}+A A^{*} f_{n+1}=A A^{*}\left(F e^{-i \arg f_{n}}\right) .
$$

In the last case, one can use the iterative process

$$
f_{n+1}=-1 / t A A^{*}\left(f_{n}-F e^{-i \arg f_{n}}\right),
$$

but the convergence domain of this process is limited.

In accordance with the procedure used in Subsection 2.3, the solutions to the nonlinear equations (43), (46), and (47) are the stationary points of the respective functionals. Since the used functionals are nonconvex, the several solutions can appear, what corresponds to existence of several local minima or saddle points. The number of solutions can vary depending on the physical parameters of the problem what requires the special careful analysis of the obtained solutions [11].

\section{Application to specific antennas}

\subsection{Amplitude-phase synthesis of cylindrical antenna}

In this Subsection, we consider the application of variational approach to the synthesis problem of cylindrical curvilinear antenna. Functional (34) is used as criterion of optimization, complex function $I$ is optimizing function. Since both the amplitude and phase of complex function $I$ are optimizing parameters, the considered problem is the amplitude-phase synthesis problem.

\subsubsection{The integral equation approach}

Let the generatrix of the antenna has the length $2 l$ and be parallel to $\mathrm{Oz}$ axis, and form of cross-section be determined by close curve $S$ which be described by formula $r=r\left(\varphi^{\prime}\right)$, where $\varphi^{\prime}$ is the angular coordinate on $S$.

In many practical applications, the antennas with currents linearly polarized along $O z$ axis are of interest. For such case, the RP has only the component $f_{\theta}(\theta, \varphi)$, denote it as $f(\theta, \varphi)$. On account of formula (18):

$$
f(\theta, \varphi)=\int_{S} \int_{-l}^{l} I\left(r\left(\varphi^{\prime}\right), z\right) \sin \theta e^{i k\left[r\left(\varphi^{\prime}\right) \sin \theta \cos \left(\varphi-\varphi^{\prime}\right)+z \cos \theta\right]} d S_{\varphi^{\prime}} d z .
$$

Let the current distribution in antenna surface be determined as

$$
I(r, z)=I_{1}\left(r\left(\varphi^{\prime}\right)\right) \cdot I_{2}(z),
$$

then the spatial RP is completely determined by the RP $f_{1}(\theta, \varphi)$, created by the distribution of current $I_{1}\left(r\left(\varphi^{\prime}\right)\right)$ in $S$, and RP $f_{2}(\theta)$ in the longitudinal plane, created by the current distribution in the generatrix of cylinder

$$
f(\theta, \varphi)=f_{1}(\theta, \varphi) \cdot f_{2}(\theta) .
$$


Function $f_{2}(\theta)$ is the RP of a linear antenna with length $2 l$. Function

$$
\left.f_{1}(\theta, \varphi)=\int_{S} I_{1} r\left(\varphi^{\prime}\right), z\right) \sin \theta e^{i k\left[r\left(\varphi^{\prime}\right) \sin \theta \cos \left(\varphi-\varphi^{\prime}\right)\right]} d S_{\varphi^{\prime}}
$$

is the RP of a plane curvilinear antenna with form $S$. Consequently, one can reduce the synthesis problem of cylindrical antenna to two independent problems for synthesis of linear and plane curvilinear antennas.

There is a many literature sources on the synthesis problem of linear antennas (see, e.g. [9] and references there). Therefore we consider here the synthesis problem for plane curvilinear antenna.

It is easily seen from formula (53) that the angle $\theta$ determines effective electrical scale of antenna. Therefore one can suppose $\theta=\pi / 2$. Omitting the indices " 1 "' in the distribution of current and RP, we represent the RP (56) in form

$$
f(\varphi)=A I:=\int_{0}^{2 \pi} I\left(\varphi^{\prime}\right) e^{i k\left[r\left(\varphi^{\prime}\right) \cos \left(\varphi-\varphi^{\prime}\right)\right]} \sqrt{r^{2}+\left(d r / d \varphi^{\prime}\right)^{2}} d \varphi^{\prime},
$$

that is, RP is determined due to action of linear bounded operator $A$.

The amplitude-phase synthesis problem for closed plane curvilinear antenna according to desired amplitude RP F consists of determination of such distribution of the current $I\left(\varphi^{\prime}\right)$, that the amplitude RP $|f(\varphi)|$ created (synthesized) by it, is the most close to $F(\varphi)$. The functional (34) is used as the criterion of optimization. The inner products in the spaces of the RPs and currents are defined as

$$
\begin{gathered}
\left(f_{1}, f_{2}\right)_{f}=\int_{0}^{2 \pi} p(\varphi) f_{1}(\varphi) f_{2}^{*}(\varphi) d \varphi, \\
\left(I_{1}, I_{2}\right)_{I}=\int_{0}^{2 \pi} I_{1}\left(\varphi^{\prime}\right) I_{2}^{*}\left(\varphi^{\prime}\right) \sqrt{r^{2}+\left(d r / d \varphi^{\prime}\right)^{2}} d \varphi^{\prime} .
\end{gathered}
$$

Using (47), (57), and definitions (58) and (59), we receive the nonlinear equation with respect to RP $f$

$$
t f(\varphi)+\int_{0}^{2 \pi} p\left(\varphi_{1}\right) K\left(\varphi, \varphi_{1}\right) f\left(\varphi_{1}\right) d \varphi_{1}=B f,
$$

where $K\left(\varphi, \varphi_{1}\right)$ is the kernel of operator $A A^{*}$

$$
K\left(\varphi, \varphi_{1}\right)=\int_{0}^{2 \pi} e^{i k r\left(\varphi^{\prime}\right)\left[\cos \left(\varphi-\varphi^{\prime}\right)-\cos \left(\varphi_{1}-\varphi^{\prime}\right)\right]} \sqrt{r^{2}+\left(d r / d \varphi^{\prime}\right)^{2}} d \varphi^{\prime},
$$

and nonlinear operator $B$ is determined as

$$
B f=\int_{0}^{2 \pi} p\left(\varphi_{1}\right) K\left(\varphi, \varphi_{1}\right) F\left(\varphi_{1}\right) e^{i \arg f\left(\varphi_{1}\right)} d \varphi_{1} .
$$


The methods of successive approximations are applied for solving the nonlinear equation (60). The simplest of them

$$
f_{n+1}=1 / t\left(B-A A^{*}\right) f_{n}
$$

has the limited region of convergence determined by formula

$$
t>2 \sqrt{2 \pi} l\left(\int_{0}^{2 \pi} p^{2}(\varphi) d \varphi\right)^{1 / 2},
$$

where $l$ is the length of contour $S$. Once the function $f$ is found, the optimal current $I$ is determined by formula (48).

The iterative process (51) is more preferable, it yields the converging sequence of functional $\sigma_{t}$ which satisfies the condition

$$
\sigma_{t}\left(f_{n+1}\right)<\sigma_{t}\left(f_{n}\right)
$$

for arbitrary $t$.

\subsubsection{The gradient methods of optimization}

Above we mentioned the methods of successive approximations for solving the arising nonlinear equations. The direct optimization of $\sigma_{t}$ functional by the gradient methods can be also applied for solving the synthesis problem. The simplest gradient method is defined by the formula

$$
I_{n+1}=I_{n}+\delta_{n} z_{n}
$$

where $\delta_{n}$ is an optimizing multiplier, $z_{n}$ is a gradient of functional $\sigma_{t}(34)$ on the function $I_{n}$ :

$$
z_{n}=A^{*}\left(f_{n}-F e^{i \arg f_{n}}\right)+t I_{n}
$$

and

$$
\delta_{n}=\left\|z_{n}\right\| / a_{n}
$$

where $a_{n}$ is a number determined by known values in the $n+1$-th iteration [4].

The disadvantage of method (66) is that only the information about optimizing function from previous iteration is used, in addition it has the slow convergence at the end of iterative process. The method of conjugate gradients [28]

$$
I_{n+1}=I_{n}+\delta_{n} h_{n}
$$

and proposed in [3] generalized gradient method

$$
I_{n+1}=\sum_{m=1}^{M} \delta_{n}^{(m)} r_{n}^{(m)},
$$

do not have such disadvantage. Here $h_{n}$ is a combination of $z_{n}$ from previous iterations, and $r_{n}^{(1)}=I_{n}, r_{n}^{(2)}=z_{n}, r_{n}^{(3)} \ldots, r_{n}^{(M)}$ is a set of some orthogonal functions, $\delta_{n}$ and $\delta_{n}^{(m)}$ are the coefficients subject to determination. 
For method (70), the problem of minimization of $\sigma_{t}$ is reduced to the solution of nonlinear algebraic system

$$
\sum_{m=1}^{M}\left[t\left(r_{n}^{(m)}, r_{n}^{(j)}\right)_{I}+\left(A r_{n}^{(m)}, A r_{n}^{(j)}\right)_{f}\right] \delta_{n}^{(m)}=\left(F e^{i \arg f_{n+1}}, A r_{n}^{(j)}\right)_{f},(j=1, \ldots, M)
$$

with unknown $\delta_{n}^{(m)}$. This system is solved effectively by the method of successive approximations substituting in its right hand side the function $\arg f_{n}$ from previous iteration. Such iterative process is converging and similarly to iterative process (51) yields the converging sequence of $\sigma_{t n}$ which satisfies the condition (65).

\subsubsection{The numerical results}

The numerical results are shown for the prescribed amplitude $\operatorname{RPs} F(\varphi)=\sin ^{2}(\varphi / 2)$ and $F(\varphi)=\sin ^{128}(\varphi / 2)$ (Fig. 1a and Fig. 1b respectively). The influence of the parameter $t$ in the functional (34) on the quality of synthesis is investigated. One can see that decrease of $t$ improves the proximity of given $F$ and synthesized $|f|$ RPs. But the norm ||$I||$ of currents grows if $t$ decreases. In the case of narrow $F$ it is necessary to diminish $t$ in order to decrease the mean-square deviation of the RPs. The detailed information about the synthesis quality is shown in Table 1.

\begin{tabular}{llllll} 
& \multicolumn{2}{l}{$F(\varphi)=\sin ^{2}(\varphi / 2)$} & \multicolumn{3}{c}{$F(\varphi)=\sin ^{128}(\varphi / 2)$} \\
$t$ & $\sigma$ & $\mathcal{K}$ & $t$ & $\sigma$ & $\kappa$ \\
0.1 & 0.0024 & 0.3419 & 0.01 & 0.0411 & 0.3450 \\
1.0 & 0.1437 & 0.4367 & 0.10 & 0.0612 & 0.3581 \\
10.0 & 1.2744 & 1.1065 & 1.0 & 0.0977 & 0.4272 \\
\hline
\end{tabular}

Table 1. The values of $\sigma$ and $\kappa$ for two desired $\operatorname{RPs} F(\varphi)$

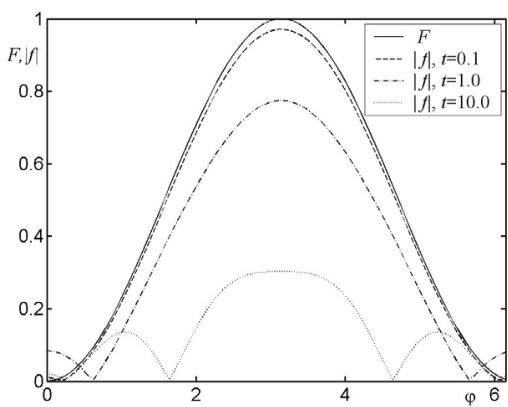

(a) $F(\varphi)=\sin ^{2}(\varphi / 2)$

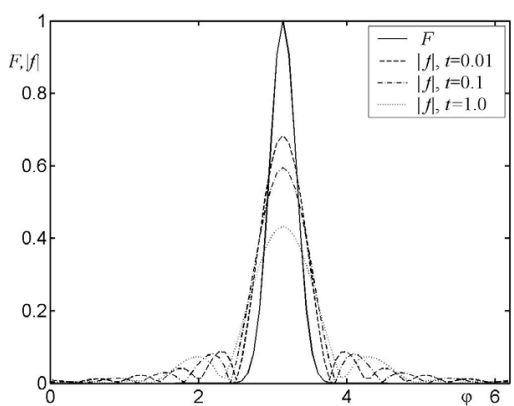

(b) $F(\varphi)=\sin ^{128}(\varphi / 2)$

Figure 1. Dependence of synthesis quality on the parameter $t$ in the functional $\sigma_{t}$

\subsection{The problem of amplitude synthesis for resonant antennas}

Resonant antennas are a new type of antennas [24], which allow to form the radiation characteristics satisfying a wide spectrum of practical requirements. Such antennas 
are formed by several surfaces, one of which is semitransparent. Antennas with one semitransparent and other metal boundary are considered here.

The synthesis problem consists of determination of such parameters of antenna (the geometry of inner boundary and transparency of the outer boundary), which form the amplitude RP or front-to-rear factor (FRF) the most close to the prescribed ones.

\subsubsection{Generalization of variational statement}

The generalized method of eigen oscillations [1] is the mathematical basis for solving the analysis (direct) problem of resonant antennas. The two-dimensional model of antennas (the case of $E$-polarization) is considered.

The main constructive parameter of resonant antennas is the cophased field in the outer surface. This field can be considered quite real (i.e., only its amplitude can be considered) since the constant phase shift of field does not change the amplitude RP. In this connection, the synthesis problem for resonant antennas is formulated as the amplitude synthesis problem.

The direct problem consists of determination of the RP $f(\varphi)$ by the known field $v(S)$ in the outer boundary $S$ of the given form. The RP created by this field can be presented similarly to (26). The operator $A$ in the case of circular external boundary has form [37]

$$
A v=\int_{0}^{2 \pi} K\left(\varphi, \varphi^{\prime}\right) v\left(\varphi^{\prime}\right) d \varphi^{\prime},
$$

where kernel

$$
K\left(\varphi, \varphi^{\prime}\right)=\sum_{n=0}^{\infty} \frac{i^{n} \cos n\left(\varphi-\varphi^{\prime}\right)}{\left(1+\delta_{0 n}\right) H_{n}^{2}(k a)},
$$

$\delta_{0 n}$ is the Kronecker delta, function $H_{n}^{2}(k a)$ is the Hankel function of second kind, $a$ is the circle radius.

In the case of resonant antenna with arbitrary outer boundary, the method of auxiliary sources $[2,12,18]$ is used for determination of the RP $f$ by the field $v$. In this method, the field $u(r, \varphi)$ outside of antenna is represented approximately by the finite sum

$$
u(r, \varphi)=\sum_{n=1}^{N} a_{n} H_{0}^{2}\left(k R_{n}\right),
$$

where $R_{n}=\sqrt{r^{2}+r_{n}^{2}-2 r r_{n} \cos \left(\varphi-\varphi_{n}\right)}$ is the distance between an observation point and $n$-th auxiliary source; $r, \varphi$ and $r_{n}, \varphi_{n}$ are the polar coordinates of a point of observation and $n$-th source, respectively; $a_{n}$ are the unknown coefficients subject to determination in the process of solving the synthesis problem.

The RP is given by

$$
f(\varphi)=\sqrt{\frac{2}{\pi}} e^{i \pi / 4} \sum_{n=1}^{N} a_{n} e^{i k r_{n} \cos \left(\varphi-\varphi_{n}\right)} .
$$


Solving the synthesis problem, we determine the field in outer boundary $S$ of the antenna and transparency of this boundary. The form of inner boundary $S_{0}$ is determined as a curve of constant phase of the field $u(r, \varphi)$ [37].

Additionally, the restrictions on a field in some areas of a near zone can be prescribed. The functional

$$
\sigma_{t}=\int_{0}^{2 \pi} p(\varphi)[F(\varphi)-|f(\varphi)|]^{2} d \varphi+\sum_{i=1}^{M} \int_{S_{i}} p_{i}\left(S_{i}\right)\left[U_{i}\left(S_{i}\right)-\left|u_{i}\left(S_{i}\right)\right|\right]^{2} d S_{i}+t \int_{0}^{2 \pi} v^{2}(\varphi) d \varphi,
$$

which is generalization of (34), allows to take into account these requirements. Here $U_{i}\left(S_{i}\right)$ are prescribed values of the field's amplitude in the areas of restriction, $u_{i}\left(S_{i}\right)$ are the obtained values of the field. Functions $p(\varphi), p_{i}\left(S_{i}\right)$ are the weight functions, allowing to adjust a degree of proximity of the given and received values of RP and field, $t$ is the parameter limiting norm of the field $v$.

In the first step of solving the synthesis problem, the field $v$ on the outer boundary $S$ is determined from a condition of minimum of the functional (76). Minimization of functional can be carried out by the gradient methods, or by solving the respective Lagrange-Euler's equation. In the first case, the generalized gradient method [3]

$$
v_{n+1}=\delta_{n}^{(1)} v_{n}+\delta_{n}^{(2)} z_{n}+\delta_{n}^{(3)} h_{n}
$$

is used. The gradient $z$ of the functional (76) (by virtue of the requirement of real field) has form

$$
z=t v-\operatorname{Re} A^{*}[F \exp (i \arg f)-f]-\sum_{i=1}^{M} \operatorname{Re} B_{i}^{*}\left[U_{i} \exp \left(i \arg u_{i}\right)-u_{i}\right],
$$

where $A^{*}$ and $B_{i}^{*}$ are the operators adjoined to $A$ and $B_{i}$, respectively [4]. Each step of iterative process (77) reduces $\sigma_{t}$. Since $\sigma_{t}$ is limited from below $\left(\sigma_{t} \geq 0\right)$, the process (77) is converging.

In the second step, the transparency $\rho$ of the outer boundary $S$ and the form of inner metal boundary $S_{0}$ are determined.

For the antenna with circular outer boundary, the transparency distribution can be presented in the explicit form

$$
\rho(\varphi)=\pi k v(\varphi) /\left(2 \sum_{n=0}^{N} \frac{a_{n} \cos n \varphi}{J_{n}^{2}(k a)+N_{n}^{2}(k a)}\right),
$$

where $J_{n}$ and $N_{n}$ are the Bessel and Neumann functions, respectively.

In the case of antenna with arbitrary outer boundary, similarly to [37], the distribution of transparency is determined by the formula

$$
\rho(\varphi)=1 /\left[\frac{\partial \psi(S(\varphi))}{\partial N}\right]
$$

\subsubsection{The numerical results}

The numerical calculations are carried out for the resonant antenna with a given outer elliptic boundary. The prescribed amplitude RP is: $F(\varphi)=\sin ^{8}(\varphi / 2)$. In Fig. 2, the results are presented for the antenna with parameters $k b=15$ and different $k a: k a=12.75, k a=14.25$, 
where $b$ and $a$ are the big and small semiaxes of ellipse. For such antenna the level of side lobes in the synthesized amplitude RP $|f|$ is smaller than $-20 \mathrm{~dB}$, and distribution of transparency is smoother in the area of main radiation (the continuous lines in Figs. $2 \mathrm{a}, 2 \mathrm{~b}$ correspond to $k a=12.75$, and the dashed ones correspond to $k a=14.25)$. The outer elliptic boundaries (dashed lines), the found form of inner metallic boundaries (continuous lines), and the inner contour $S_{a}$ of placement of the auxiliary sources (dash-and-dot lines) are shown in Figs. 3a, 3b. The auxiliary sources are distributed uniformly on the $S_{a}$.

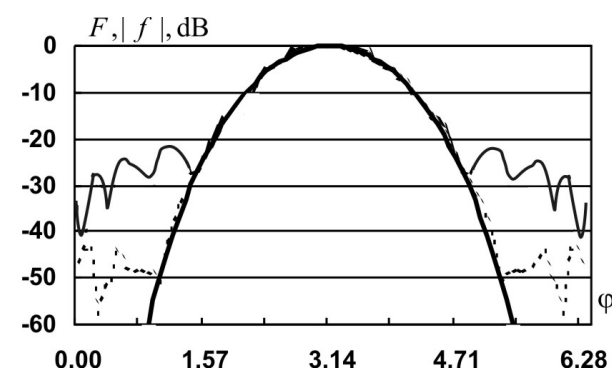

(a) the prescribed $F$ (thick line) and synthesized RPs $|f|$

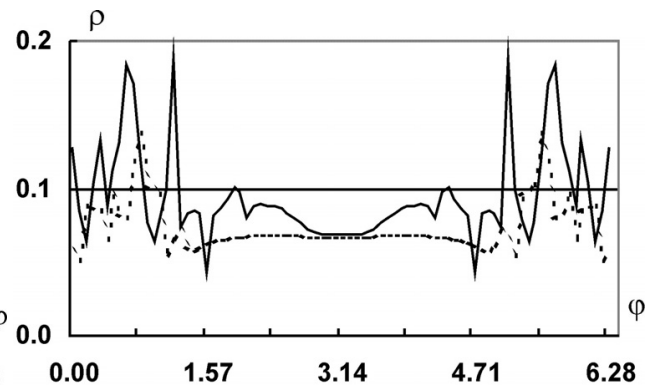

(b) the transparency $\rho$ of outer boundary $S$

Figure 2. Synthesis of resonant antenna with elliptic outer boundary

The distribution of transparency $\rho$ in the area opposite to direction of main radiation has a spasmodic character. Such distribution cannot be realized by the physical reason. Therefore the values of $\rho$ are averaged in this range in order to receive the smooth distribution of $\rho$. This leads to some change of field $v$ on $S$, but the numerical calculations show small change of the synthesized amplitude RP $|f|$. The more smooth distribution of $\rho$ in the area mentioned above can be achieved by increasing the number of auxiliary sources here.

The numerical results for solution of the synthesis problem with restrictions on the field in a near zone are given for the antenna with circular outer boundary. The prescribed amplitude $\mathrm{RP}$ is: $F(\varphi)=\sin ^{8}(\varphi / 2) ; k a=15$. Minimization of a field was carried out in two points $\varphi=\pi / 2,3 \pi / 2$ on the additional circle with radius $k b=20$. These points were allocated in the second summand of the functional (76) using the weight function $p_{1}(\varphi)=\delta(\pi / 2,3 \pi / 2)$; $p(\varphi) \equiv 1, t=0.01$. In Fig. 4a, the prescribed RP $F$ (thick continuous line) and synthesized $|f|$ (thin continuous line) amplitude RP are shown. The amplitude $\left|u_{1}\right|$ of obtained field on the circle of restrictions is marked by dashed line.

It can be seen that the field at restriction points is reduced up to level $-37 \mathrm{~dB}$. The synthesized field $v$ (continuous line) and transparency $\rho$ (dashed line) are presented in Fig. $4 \mathrm{~b}$. The form of the inner synthesized metallic boundary is more complicate than in the previous example.

\subsection{Waveguide resonant antenna}

Synthesis of resonant antenna with waveguide excitation is carried out according to the FRF. The optimizing functional enables to take into account a various requirements to the FRF of antenna in the operating frequency range, as well as outside this range. 


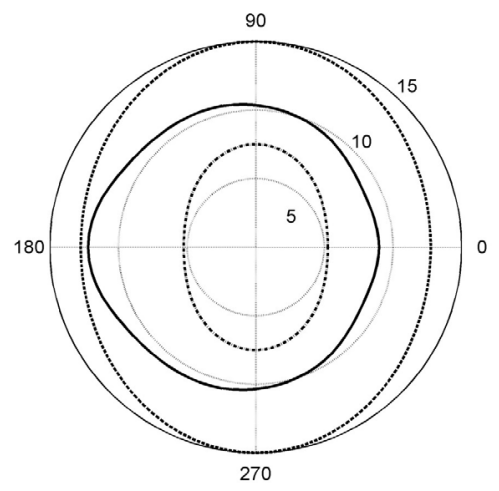

(a) $k a=12.75$

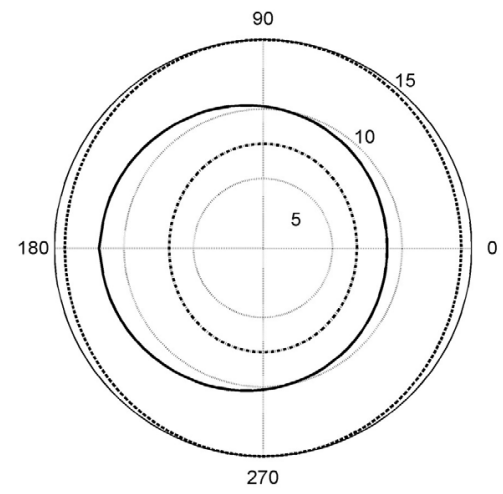

(b) $k a=14.25$

Figure 3. Form of antenna boundaries $S$ and $S_{0}$

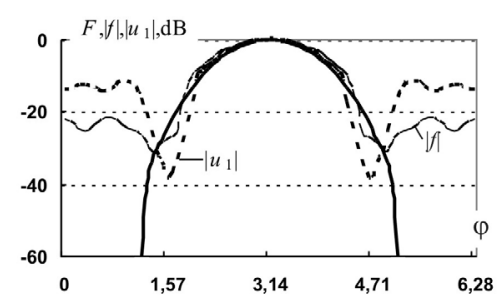

(a) the RPs and field in area of restriction

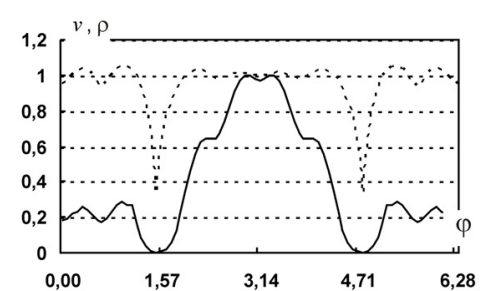

(b) the field $v$ and transparency $\rho$ in outer boundary

Figure 4. Synthesis of resonant antenna with restriction on the field

\subsubsection{The physical description of problem}

The geometrical parameters of resonant antenna with waveguide excitation are shown in Fig. 5. In order to create RP enough narrow, the width $L$ of antenna should be much larger than the wavelength $\lambda$. The height $d$ is of the order of $\lambda / 2$. Excitation is carried out by a metal single-mode waveguide with semitransparent grid at its end; the width $l$ of waveguide is of the order $\lambda / 2$, and both its length and the length $D$ of the antenna along the $O x$ axis are of the order of $L$.

The RP of antenna has form

$$
f(\theta, \varphi)=\iint_{S} u(x, y) \exp [i k(x \sin \theta \cos \varphi+y \sin \theta \sin \varphi)] d x d y .
$$

The direct (analysis) problem on determination of the electromagnetic field components in the semitransparent aperture is reduced to two separate problems in the planes $x \mathrm{Oz}$ and $y \mathrm{Oz}$ respectively. We consider here the case of E-polarization. The unknown function $u$ is the $E_{y}$ 


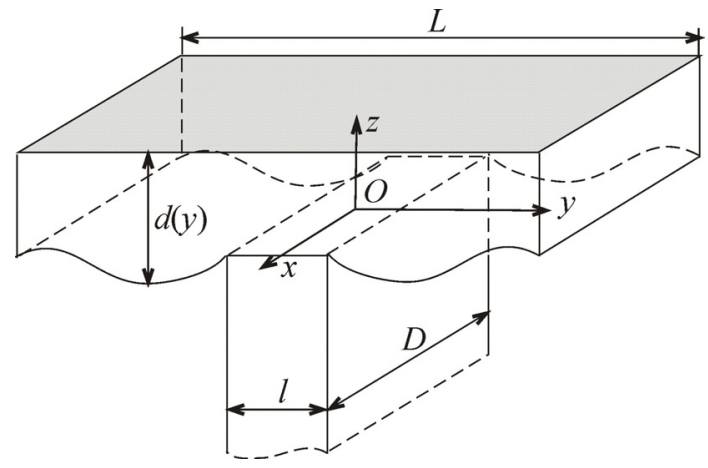

Figure 5. Geometry of the resonant antenna

component of electromagnetic field. In the region over the antenna this component satisfies the Helmholtz equation and the boundary conditions

$$
u=0
$$

on the all metallic walls;

$$
u^{+}=u^{-}, \quad \frac{\partial u^{-}}{\partial x}-\frac{\partial u^{+}}{\partial x}=\frac{u}{\rho(S)}
$$

on the semitransparent upper boundary, the same conditions in the aperture of the exciting waveguide; the condition of radiation on the infinity

$$
u_{\substack{r \rightarrow \infty \\ x>0}}=\frac{\exp (-i k r)}{\sqrt{k r}} f(\varphi),
$$

and asymptotical condition in the exciting waveguide

$$
u_{\substack{x \rightarrow-\infty \\ y+<a}}=\cos \frac{\pi y}{2 a}\left(\exp \left(-i \beta_{1} x\right)+R_{1} \exp \left(i \beta_{1} x\right)\right) .
$$

The problem of determination of the field $u(x, y)$ in the semitransparent boundary $S$ is solved in three steps [37]. In the first step, the field in the irregular region of antenna is determined using the cross-section method [19]. In the second step, the field over the exciting waveguide is sought for, and the matching of field in the regular and irregular regions of antenna is fulfilled. The reflection coefficient $R_{1}$ is determined by the fulfillment of the adjoint boundary conditions (85) in the third step.

Under condition of the linear polarization of field in the aperture of exciting waveguide, the $\mathrm{RP}(81)$ can be represented as product of two functions, namely the RP of plane antenna with variable height $d(y)$ of the upper wall and transparency $\rho(y)$ of the lower wall, and RP of the linear antenna in the $x \mathrm{Oz}$ plane. 
In such a way, the RP in the $y \mathrm{Oz}$ plane can be written down in the form [37]

$$
f(\theta, \varphi)=k \sin \theta \int_{-L / 2}^{L / 2} u(y, 0) \exp (i k y \sin \varphi) d y .
$$

Numerical calculations can be essentially simplified, if one assumes that the field above the antenna is represented approximately in the form [37]

$$
u_{\mid \begin{array}{c}
|y|<L / 2 \\
z \rightarrow+0
\end{array}} \cong u(y, 0) \exp (-i k z) .
$$

\subsubsection{The objective functionals}

In the process of statement of the synthesis problem one requires to provide the best approximation to the prescribed FRF in the operating frequency range $\left[k_{1}, k_{2}\right]$ and the minimal values of the FRF outside this range, that is for $k \in\left[k_{0}, k_{1}\right)$ and $k \in\left(k_{2}, k_{3}\right]$.

The variational approach for solving this problem was developed in [37]. Modification of variational statement of the synthesis problem is proposed. The problem consists of determination of functions $d(y)$ and $\rho(y)$, which maximize FRF $\eta_{1}$ in the operating range $\left[k_{1}, k_{2}\right]$. In this case, the least value of FRF in this range is specified as a criterion of optimization, and this value is maximized by a choice of functions $d$ and $\rho$. That is, the functional is maximized

$$
\eta_{1}=\max \min _{k_{1} \leq k \leq k_{2}} \eta(k)
$$

The additional parameter of optimization $1-\left|R_{1}(k)\right|^{2}$, where $R_{1}(k)$ is the reflection factor of the main wave in exciting waveguide, with some weight multiplier can be included into functional (88). In this case, the transparency of waveguide aperture can be also used as an additional parameter of optimization.

Minimization of FRF outside the operating frequency range is one of requirements of electromagnetic compatibility for radiating systems [36]. Thus, the value of FRF should remain the largest in the main frequency range. Under these requirements, the following generalization of the variational statement of problem is considered: to find functions $d$ and $\rho$, maximizing the functional $\eta_{1}$, and, at the same time, minimizing additionally the following functional [6]

$$
\eta_{2}=\min \max _{k_{0} \leq k<k_{1}, k_{2}<k \leq k_{3}} \eta(k),
$$

that is, minimization of the maximal FRF value outside the $\left[k_{1}, k_{2}\right]$ range is required. Of course, it is necessary to take into account the restrictions on the functions $d(x)$ and $\rho(x)$ owing the physical reason:

$$
d_{0}<d(y)<d_{m}, \quad \rho_{0}<\rho(y)<\rho_{m} .
$$

For example, $d_{0}, d_{m}$ are the boundary values of height, which provide the single-mode conditions in antenna, and $\rho_{0}, \rho_{m}$ are the boundary values of transparency, which provide a good quality of antenna in the required ranges. Moreover, the received functions should 
satisfy the additional conditions of smoothness, which can be formulated as restriction on the second derivatives

$$
\left|d^{\prime \prime}(y)\right| \leq M_{1}, \quad\left|\rho^{\prime \prime}(y)\right| \leq M_{2}
$$

\subsubsection{The modeling results}

The numerical results are shown in Fig. 6. Calculations were carried out for the problem of $\eta$ maximization in range $\pm 5.0 \%$ in neighborhood of the central frequency $\left(k_{c}=6.0\right)$. It is necessary to minimize the FRF outside of this range for $5.2<k<5.6$ and $6.4<k \leq 6.8$. Parameters of antenna are the following: the length of antenna $L=6.0$, half-width of excited waveguide $l / 2=0.3 L$, number of considered reflected waves in waveguide $N_{1}=5$, number of waves in a background $N_{2}=20$.

The optimized values of FRF are marked by solid line in the basic and additional ranges; the dashed line corresponds to not optimized values of $\eta$ in the main and additional ranges.

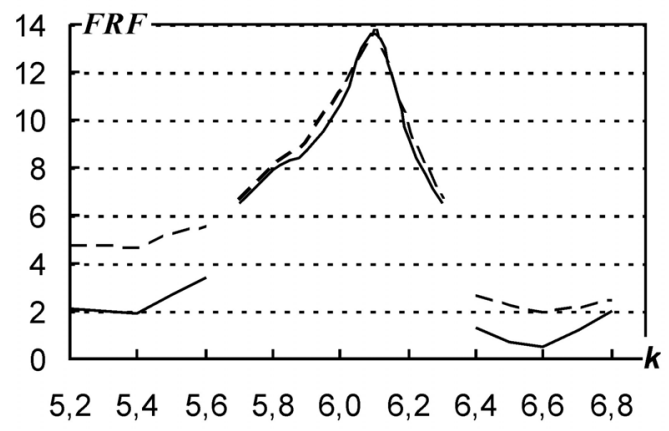

Figure 6. Optimized values of FRF in the basic and additional ranges versus the frequency $(k)$

Process of additional optimization is carried out on the simplified procedure, that is the control of decrease of the FRF in the basic range is omitted [6]. Therefore, the values of FRF in the basic range are slightly decreased in comparison with the FRF values for initial problem.

The optimal form $d(y)$ of the lower boundary of antenna and transparency $\rho(y)$ of the upper boundary are slightly different for the both cases of optimization.

In Fig. 7, the change of the FRF values at three points of main and additional ranges of frequency (two extreme points and middle one) is shown. The width of main range is equal to $8.33 \%$, and width of additional range is equal to $10.0 \%$. In Fig. 7a, curve 1 corresponds to the central value of frequency $k=12.0$, and curves 2 and 3 correspond to the extreme points $k=11.0$ and $k=13.0$ respectively. In Fig. $7 \mathrm{~b}$, curve 1 corresponds to the central frequency $k=14.0$, and curves 2 and 3 correspond to the frequency values $k=13.01$ and $k=15.0$. One can see that the main optimization takes place in the first iterations; there is the improvement only in low-order digit in the next steps. Therefore, it is enough to make 3-5 steps for the main range and 9-11 steps for the additional range, in iterative procedure to receive the practically interesting results.

The total number of iterations also depends on the width of the considered frequency ranges. 


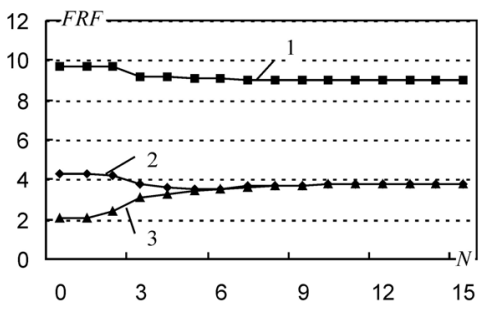

(a) the main range

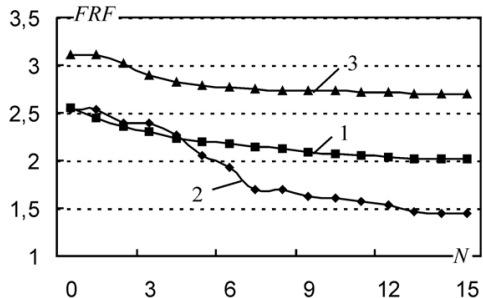

(b) the additional range

Figure 7. The FRF values versus the iteration number $N$

\subsection{Phase synthesis problem for cylindrical array}

The phase distributions of excitation currents in the array's elements are the optimizing parameters in the problem of phase synthesis. The optimization of considered functionals is reduced to the solution of the corresponding system of nonlinear equations. The gradient methods for direct optimization of functionals are used in practical applications.

\subsubsection{The RP of array}

A series of simplifications in process of synthesis of the cylindrical array is used in order to reduce the computing time $[9,22]$. Separation of variables onto the vertical and horizontal components for the distributions of currents in the array elements, as well as for the RPs, is one of the simplifications.

Thus, the expressions for current $I_{n m}(x, y)$ in radiators and RP $f(\theta, \varphi)$ have the form [4]

$$
\begin{gathered}
I_{n m}(x, y)=I_{n m}^{1}(x) \cdot I_{n m}^{2}(y), \\
f(\theta, \varphi)=f_{1}(\theta) \cdot f_{2}(\varphi) .
\end{gathered}
$$

It is assumed that the radiating elements are flat apertures, for example, end of open waveguide. The RP depends on the angular coordinates $\theta$ and $\varphi$. From the practical point of view, the consideration of such models of the arrays is justified by the fact that they allow to receive the values of required radiation characteristics with the accuracy of $2 \%-$ $5 \%$, while the time of solution for the respective problems of analysis (determination of the $\mathrm{RP}$ of array), considerably decreases. Such approach to the solution of direct electrodynamic problems is effective especially for the arrays with constant coordinate surfaces, e.g., for the plane, cylindrical and conical arrays. Proceeding from the above assumptions, we separate the synthesis problem of such arrays into two synthesis problems for the linear and circular arrays.

The spatial RP of cylindrical array [9] is:

$$
f(\theta, \varphi)=\sum_{m=1}^{M} \sum_{n=1}^{N} I_{n m} f_{n m}(\theta, \varphi) \exp \left(i k\left(z_{m} \cos \theta+a \sin \theta \cos \left(\varphi-\varphi_{n}\right)\right)\right),
$$

where $N$ is the number of radiators in circular subarray (identical for all subarrays), $M$ is the number of circular subarrays, $a$ is radius of cylinder. The currents $I_{n m}$ are complex numbers, by means of which choice the approximation to the given amplitude $\operatorname{RP} F(\vartheta, \varphi)$ is carried out. 
The functions $f_{n m}(\vartheta, \varphi)$ are the RPs of separate radiators, $\left(z_{m}, a, \varphi_{n}\right)$ are the coordinates of radiators. The $\mathrm{RP}$ of separate radiators is identical and can be presented in the following form

$$
f_{n m}(\theta, \varphi)=f_{n}\left(\varphi-\varphi_{n}\right) f_{m}(\theta) .
$$

Following the above assumptions, the RP (94) can be written as

$$
f(\theta, \varphi)=f_{l}(\theta) f_{c}(\varphi),
$$

where

$$
f_{l}(\vartheta)=A_{1} I:=\sum_{m=1}^{M} I_{m} f_{m}(\vartheta) \exp \left(i k z_{m} \cos \vartheta\right)
$$

is the RP of linear array, and

$$
f_{c}(\theta, \varphi)=A_{2} I \equiv \sum_{n=1}^{N} I_{n} f_{n}\left(\varphi-\varphi_{n}\right) \exp \left(i k a \sin \theta \cos \left(\varphi-\varphi_{n}\right)\right)
$$

is the RP of circular array for each $\theta$. Below we consider the synthesis problem for the circular array.

\subsubsection{Optimization criteria}

The complex currents in the array elements are determined by their amplitudes and phases. We denote these values $\left|I_{n}\right|$ and $\psi_{n}$, respectively. The amplitudes $\left|I_{n}\right|$ of currents are prescribed together with the amplitude RP $F$ in the problem of phase synthesis. The phases $\psi_{n}$ are the optimizing parameters. We use the functionals (29) and (33) for optimization.

The equalities

$$
\begin{aligned}
& \chi=\arg A\left(|I| e^{i \psi}\right), \\
& \psi=\arg A^{*}\left(F e^{i \chi}\right) .
\end{aligned}
$$

should be satisfied at the points of functional (33) maximum. This set yields the system of transcendental equations for the phases $\psi$ of current and phase RP $\chi$.

Using normalization of the current $I$ values: $\|I\|=1$, we write down the functional (33) in two equivalent forms

$$
\kappa=\left(F e^{i \chi}, A\left(|I| e^{i \psi}\right)\right)_{f}=\left(A^{*}\left(F e^{i \chi}\right),|I| e^{i \psi}\right)_{I},
$$

where $\chi=\arg f$ is the phase RP. The operator $A^{*}$ is adjoint to $A$ and determined similarly to [4].

At first, we consider the problem of $\kappa$ maximization. Substituting (99) into (100), we receive the system of nonlinear algebraic equations for optimal phase distribution of currents

$$
\psi_{n}=\arg A^{*}\left(F \exp \left(i \arg A\left(|I| e^{i \psi}\right)\right)\right), n=1,2, \ldots, N .
$$

In practice, the system (99), (100) is more convenient for the numerical solution. For this reason, we use the following iterative process

$$
\arg f^{(k)}=\arg A\left(|I| \exp \left(i \psi^{(k)}\right)\right),
$$


to find the phases $\psi^{(k+1)}$.

$$
\psi^{(k+1)}=\arg A^{*}\left(F \exp \left(i \arg f^{(k)}\right)\right.
$$

The gradient methods are more convenient to solve the minimization problem for the functional $\sigma$. The method of conjugated gradients [28] is the most suitable for this purpose. In this method the next approximation of phase vector $\psi=\left\{\psi_{n}, n=1, \ldots N\right\}$ is calculated by the formula

$$
\psi^{(k+1)}=\psi^{(k)}+\delta^{(k)} h^{(k)} .
$$

Here

$$
h^{(k)}=z^{(k)}+\gamma^{(k)} h^{(k-1)},
$$

$z^{(k)}$ is the gradient of $\sigma$ with respect to the phases of currents in the "point" $\psi^{(k)}$. The components of vector $z^{(k)}$ are the following

$$
z_{n}^{(k)}=-\operatorname{Im}\left\{\left(A^{*}\left(\left(F-s\left|f^{(k)}\right|\right) \exp \left(i \arg f^{(k)}\right)\right)\right)_{n} \cdot I_{n}^{(k)}\right\}, n=1,2, \ldots, N .
$$

Parameter $\delta^{(k)}$ is determined from minimum of $\sigma$ being a function of this parameter.

In practice, it is necessary to solve the problem of discrete phase synthesis [9, 22], because the arbitrary phase distributions cannot be realized in the array radiators. These distributions are prescribed as a set of discrete values, which are multiple to the given phase discrete value $\Delta$, that is $\psi_{n}=\lambda_{n} \Delta$, the integers $\lambda_{n}$ are unknown in this case [5, 7].

The algorithm consisting of two enclosed iterative processes is used for the solution of this problem. The value of phase $\psi_{n}$ is improved in the $n$-th step of the internal iterative process, the phases in other radiators remain fixed. At the same time, the mean-square deviation of the synthesized RP and function $F \exp \left(i \arg f^{(k)}\right)$, where $\arg f^{(k)}$ is the phase RP, which is received in the previous step of external iterative process, is minimized. The phase RP $\arg f$ is improved in the external iterations.

The internal cycle consists of the successive improvement of phases in the separate radiators changing their number from 1 up to $N$. The value of $\sigma$ decreases in each step of internal cycle.

The new phase RP $\arg f^{(k+1)}$ is calculated by the found values of $\{\psi\}$ in external cycle. The values of $\sigma$ corresponding to new phase RP also decrease, what provides the convergence of the whole algorithm. In view of the step-type behavior of $\psi_{n}$ values, this convergence exists not only for a sequence of $\sigma$, but also for the phase distributions. The iterative process is considered completed, if there is no change of $\psi_{n}$ in the internal cycle.

The problem of discrete phase synthesis is solved in two steps. In the first step, the synthesis (with small accuracy) without the account of phase discrete values is carried out. After that, the found phases are approximated up to the nearest discrete values. In the second step, the described above algorithm of discrete synthesis is used. As a rule, one is enough to make several external cycles in the latter algorithm.

\subsubsection{The results of numerical modeling}

The numerical results are given for the sector array. The RPs of separate radiators have a cosine form, and mutual coupling of separate radiators is not taken into account [35]. The number of radiators $N=32$, the radiators are placed in active sector $\beta=90^{\circ}$. 
The synthesis results are shown in Fig. 8 for the bi-directional RP

$$
F(\varphi)=\left\{\begin{array}{c}
|\sin 18 \varphi,| \varphi \mid<5.0^{0} \\
0,|\varphi| \geq 5.0^{0}
\end{array}\right.
$$

The thick line corresponds to the prescribed amplitude RP $F$, and thin line corresponds to the synthesized RP $|f|$. The prescribed $F$ and synthesized $|f|$ RPs coincide in the main lobe up to level $-20 \mathrm{~dB}$, the level of side lobes does not exceed $-20 \mathrm{~dB}$ (see Fig. 8a).

In the practical applications, the problem of phase scanning [35] is considered for arrays. The array alongside with creating the amplitude RP which is more close to the desired one should provide the moving this $\mathrm{RP}$ along the angular coordinate in the scanning process. This moving is carried out by the change of the phase distribution $\left\{\psi_{n}\right\}$ only; the amplitudes $\left\{\left|I_{n}\right|\right\}$ of current remain constant. In fact, the problem of phase synthesis is solved for each scanning angle $\varphi_{S}$ separately.

The difference between the results of continuous and discrete synthesis depends on the value of phase discrete $\Delta$. The smaller the difference, the smaller this value. In Fig. $8 \mathrm{~b}$ the values of $\sigma$ for two types of synthesis are shown for the process of scanning, $\varphi_{s}$ is changed in range from $1^{0}$ up to $9^{0}$. Solid line corresponds to the case of continuous synthesis, and dashed line correspond to the case of discrete synthesis, the value of $\Delta=22.5^{0}$. This value of $\Delta$ gives not big difference for two types of synthesis. So, the difference between $F$ and $|f|$ in main lobe of RPs does not exceed $1 \mathrm{~dB}$, and this difference does not exceed $10 \mathrm{~dB}$ in the side lobes. Such difference is satisfactory for the engineering practice. Of course, the above mentioned difference grows if the scanning angle $\varphi_{s}$ approaches to the left or right border of the active sector $\beta$.
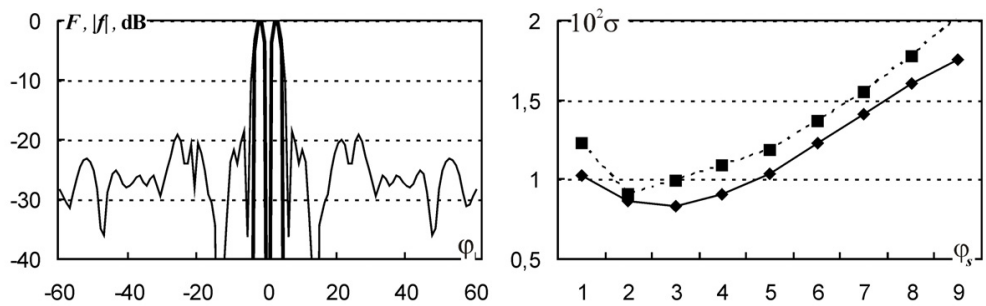

(a) the prescribed and synthesized RPs for the (b) the normalized values of $\sigma$ for the angle of scanning $\varphi_{s}=0^{0}$

continuous and discrete phase synthesis

Figure 8. Synthesis of bi-directional RP F

\subsection{Investigation of branching solution}

The problem of the non-uniqueness of solutions for phase synthesis problem is investigated on the example of linear array.

The various modifications of the Newton method have been developed for solving the nonlinear equations in [13], and have been detailed for the synthesis problems in [11]. We consider here the above approach for determination of the number of solutions and 
investigation of its properties by the example of the nonlinear equation (46), corresponding to functional $\kappa(33)$.

Let the operator $A$ describe the RP (array factor) of linear array [4].

$$
f(\xi)=A v:=\sqrt{\frac{c}{2 \pi}} \sum_{n=-M}^{M} v_{n} \exp (i c n \xi),
$$

where $c=k a \sin \alpha, a$ is the radius of array, $\alpha$ is the angle in which the RP $F$ is non-zero, $N=2 M+1$ is a total number of array elements, $\xi$ is the generalized angular coordinate. The operator $A^{*}$ acts in the following way

$$
A^{*} f=\sqrt{\frac{c}{2 \pi}} \int_{-\pi / c}^{\pi / c} f(\xi) \exp (-i c n \xi) d \xi,
$$

In this case, the functional (33) can be presented as

$$
\kappa(\psi)=\int_{-\pi / c}^{\pi / c}|f(\xi)| F(\xi) d \xi .
$$

\subsubsection{The numerical results}

The numerical calculations are carried out for the prescribed amplitude radiation pattern $F(\xi)=\cos (\pi \xi / 2)$ and are shown in Figs. 9 and 10. In Fig. 9a, the values of $\kappa$ and $\sigma$ are shown for various types of initial approximation of the current's phase $\psi^{(0)}(x)$. For this case, the maximization problem of $\kappa$ is equivalent in some sense to minimization problem of $\sigma$ [4].

The solid lines correspond to values of $\kappa$, and the dashed lines correspond to values of $\sigma$. The number of array elements $N=11$, parameter $c$ changes from $c=0$ up to $c=2$. For the values of $N c$ which do not exceed $N c=5$ all types of solutions give the same values of $\kappa$ and $\sigma$. At $N c \approx 2 \pi$ the branching of solutions appears, and optimal value for $\kappa$ and $\sigma$ functionals gives the solution with even phase $\psi$. The prescribed amplitude radiation pattern $F$ and synthesized $|f|$ are shown in Fig. 9b. The amplitudes $|f|$ in the considerable extent differ from the amplitude $F$ because of small value of $c$ parameter $(c=1.6)$.

The optimal values of sought phase distributions $\psi$ are shown in Fig. 10a, the given current amplitude distribution is $|I| \equiv 1$. The optimal phase distributions $\psi$ keep the parity properties of corresponding initial approximations $\psi_{0}$. The optimal values of $\kappa$ and $\sigma$ provide the solution with phase distribution $\psi_{0}(x)=\cos (x)$ (see Fig. 9).

The quality of approximation to prescribed amplitude pattern $F$ too much depends on the parameter $c$ (see Fig. 10b). At $c=3.14$ the $\kappa$ and $\sigma$ values are noticeably smaller than for $c=1.6$, although the value of $N$ is larger in case of Fig. 9 .

\subsection{Synthesis of waveguide array}

The mutual coupling of the separate elements of array is taken into account in the process of solution of a direct problem (analysis problem) [7, 14]. 

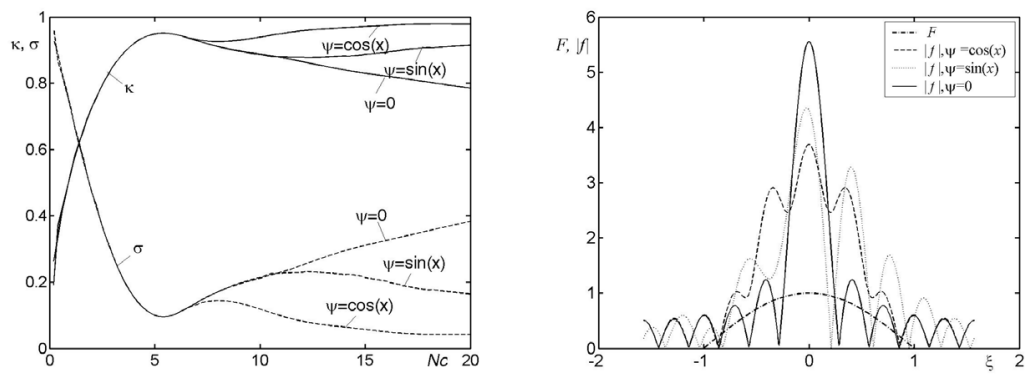

(a) the $\kappa$ and $\sigma$ values versus the array electrical (b) the synthesized amplitude radiation patterns size $N c$

for various types of current phase, $c=1.6$

Figure 9. The values of optimizing functionals and synthesized RPs for various type of solutions

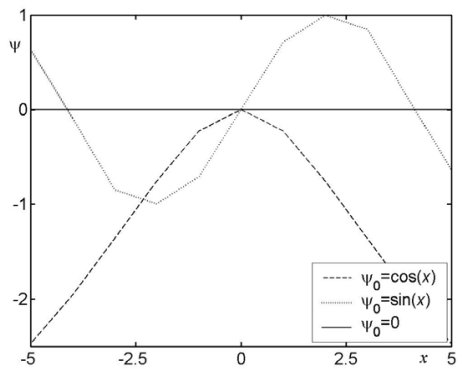

(a) the optimal phase distributions

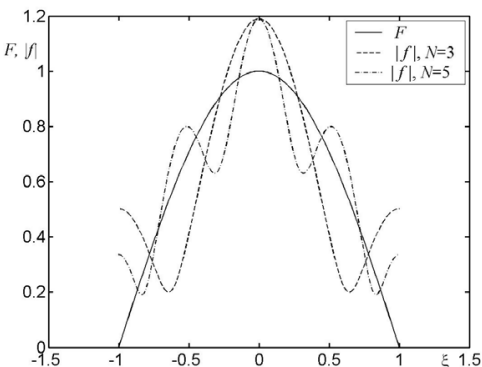

(b) the desired $F$ and synthesized $|f|$ RPs

Figure 10. The optimal phase distributions at $N=11(\mathrm{a})$, and synthesis results at $c=3.14$ (b)

\subsubsection{Statement of synthesis problem}

The objective functional is formulated as [4]

$$
\sigma=\iint_{\Omega}(F(\theta, \varphi)-|f(\theta, \varphi)|)^{2} d \theta d \varphi+t \sum_{n=1}^{N} \int_{S_{n}}\left|I_{n}\left(x_{n}, y_{n}\right)\right|^{2} d x_{n} d y_{n}
$$

where $N=2 M+1$ is a number of exciting waveguides, $F(\theta, \varphi)$ is the prescribed amplitude $\mathrm{RP},|f(\theta, \varphi)|$ is the amplitude of synthesized $\mathrm{RP}, I_{n}\left(x_{n}, y_{n}\right)$ are the currents in the waveguide apertures. Geometry of waveguide array is shown in Fig. 11.

The determination of the currents $I_{n}\left(x_{n}, y_{n}\right)$ in the waveguide apertures (the solution of analysis problem) results in solution of the integral equation system [14].

The RP (array factor) [9] of array is:

$$
f(\theta, \varphi)=\sum_{n=1}^{N} a_{n} \exp \left[i k\left(x_{n_{0}} \sin \theta \cos \varphi+y_{n_{0}} \sin \theta \sin \varphi\right)\right],
$$




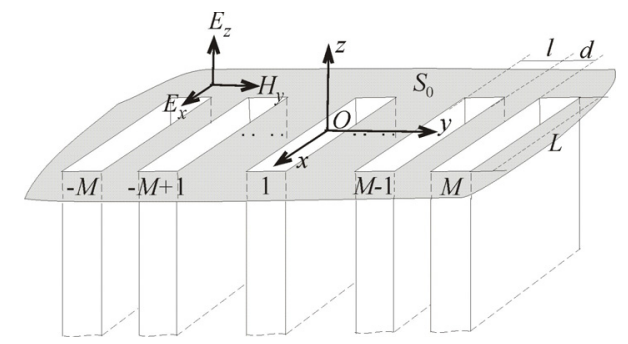

Figure 11. Geometry of plane periodical waveguide array

where $x_{n_{0}}$ and $y_{n_{0}}$ are the coordinates of central points of apertures, $a_{n}$ is the complex excitation coefficient for $n$-th waveguide.

Introducing the generalized angular coordinates $\xi_{1}$ and $\xi_{2}$, we receive the expression for the $\mathrm{RP}$

$$
f\left(\xi_{1}, \xi_{2}\right)=\sum_{n=1}^{N} a_{n} \exp \left[i k\left(x_{n_{0}} \xi_{1}+y_{n_{0}} \xi_{2}\right)\right],
$$

where

and finally

$$
\xi_{1}=\sin \theta \cos \varphi, \quad \xi_{2}=\sin \theta \sin \varphi,
$$

$$
f\left(\xi_{1}, \xi_{2}\right)=A \vec{a}:=\sum_{n=1}^{N} a_{n} f_{n}\left(\xi_{1}, \xi_{2}\right),
$$

where $\vec{a}=\left\{a_{1}, a_{2}, \ldots a_{N}\right\}, f_{n}\left(\xi_{1}, \xi_{2}\right)$ are the RPs of separate waveguides.

The expression (116) indicates that the calculation of array factor $f\left(\xi_{1}, \xi_{2}\right)$ using the excitation coefficients $a_{n}$ is realized by the linear operator $A$. The coefficients $a_{n}$ will be the optimization parameters in the synthesis problem. Solving the synthesis problem, we minimize the functional $\sigma_{t}(34)$.

\subsubsection{The modeling results}

The results of numerical calculations are presented for the waveguide arrays consisting of 15 and 31 radiators; $k l=1.2, k L=18.75, k$ is wavenumber, $l$ and $L$ are width and length of waveguide aperture respectively. The prescribed amplitude RP is: $F(\theta, \varphi)=F_{1}(\theta) F_{2}(\varphi)$, where

$$
F_{1}(\theta)=\left\{\begin{array}{l}
1,|\theta| \leq \pi / 20 \\
0,|\theta|>\pi / 20
\end{array}, F_{2}(\varphi)=(\cos \varphi)^{64}\right.
$$

In Fig. 12, the dependence of the synthesis results on the value $N$ of waveguides is shown. It is easy to see, that the synthesized amplitude RP has narrower main lobe if $N$ increases. The level of the first sidelobe is $-30.26 \mathrm{~dB}$ and $-30.71 \mathrm{~dB}$ respectively. The low level of sidelobes and velocity of its decrease is very important characteristic of the synthesized amplitude RPs. As rule, one requires the level of first sidelobe not greater than $-20 \mathrm{~dB}$ and not very slow decreasing the next sidelobes. The above mentioned characteristic for the synthesized amplitude $\mathrm{RP}$ in the plane $\xi_{2} \mathrm{Oz}$ are shown in Table 2. The amplitude $\mathrm{RP}|f|$ at $N=31$ has lower first sidelobe $-30.71 \mathrm{~dB}$ and faster decrease of the far sidelobes. 


\begin{tabular}{llllll}
\hline Sidelobe: & 1st & 2nd & 3rd & 4th & 5th \\
$N=15$ & -30.26 & -40.43 & -46.25 & -50.63 & -53.27 \\
$N=31$ & -30.71 & -40.83 & -47.52 & -52.45 & -56.33 \\
\hline
\end{tabular}

Table 2. The level of sidelobes (in $\mathrm{dB}$ ) corresponding to array with various $N$

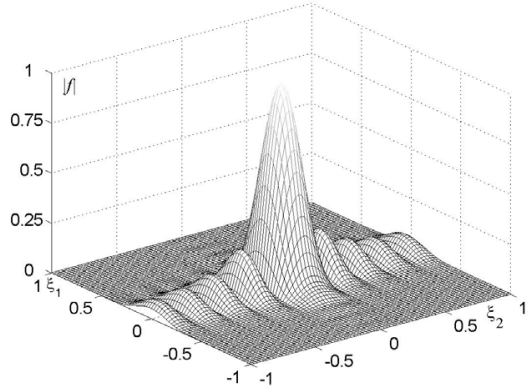

(a) $N=15$

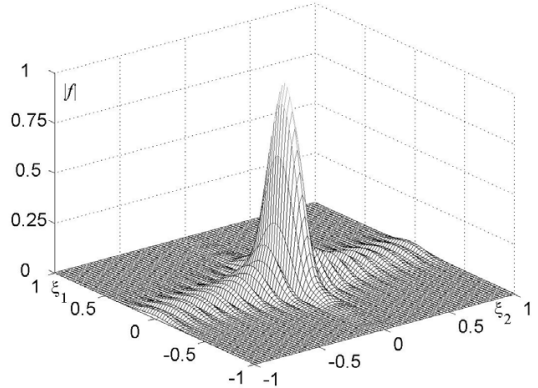

(b) $N=31$

Figure 12. The synthesized amplitude RPs for various $N$

\section{Conclusions}

In the process of solving the amplitude-phase synthesis problem, the influence of weight multiplier $t$ on the synthesis results was investigated. It turned out that the mean-square deviation of the prescribed $F$ and synthesized $|f|$ amplitude RPs diminishes if $t$ decreases. At the same time the norm $\|I\|$ of current grows. This testify that one should take into account the above fact in order to receive the solution with smaller mean-square deviation of the RPs or with small norm of current. The elaborated iterative procedures guarantee convergence of the successive approximation methods for numerical solving the arising non-linear equations.

The proposed approach for solving the synthesis problems of resonant antennas is universal, and it provides the possibility to synthesize antennas with the arbitrary form of external boundary. The calculation time of the RP of antenna is small enough what it is very important in the process of solution of the synthesis problem. The used variational statement of the synthesis problem also allows to take into account restrictions on the field at the given points (areas) of a near zone.

The synthesis of the resonant antenna with waveguide excitation gives the possibility to take into account the various requirements to the FRF in the operating frequency range. The developed algorithms enable to optimize the values of FRF in the single range, as well as in the several frequency ranges. The values of the objective parameters $d$ and $\rho$, which are received in the process of numerical calculations, are constructive characteristics of resonant antenna and they can be directly used in the antenna design.

The variational approach for solving the phase synthesis problem can be applied effectively for the plane, cylindrical and conical arrays. It allows to decrease the computational time, at the same time the accuracy of determination of the array characteristics is sufficient for practice. The branching solutions are investigated for the case of linear array. It is shown 
that one can receive the solutions with various properties starting the iterative process with different initial approximations.

The considered optimization problems of waveguide array give the possibility to take into account the requirements to the amplitude RP and amplitude-phase distribution of field in the aperture of exciting waveguides. The developed algorithms enable to achieve the minimal mean-square deviation $\sigma$ of the prescribed and synthesized amplitude RPs, and to optimize simultaneously the restrictions on the phase or amplitude characteristics of the excited fields.

\section{Author details}

Mykhaylo Andriychuk

Pidstryhach Institute for Applied Problems in Mechanics and Mathematics, NASU, Ukraine

\section{References}

[1] Agranovich, M. S., Katsenelenbaum, B. Z., Sivov, A. N., Voitovich, N. N. (1999). Generalized Method of Eigenoscillations in Diffraction Theory. Berlin, Wiley-VCH.

[2] Alexidze, M. A. (1978). Solution of Boundary Problems by Decomposition on Non-orthogonal Functions, (in Russian). Moscow, Nauka.

[3] Andriychuk, M. I., Voitovich, N. N. (1985). Synthesis of a Closed Planar Antenna with Given Amplitude Pattern. Radio Eng. and Electron. Phys., Vol. 30, No 5, pp. 35-40.

[4] Andriychuk, M. I., Voitovich, N. N., Savenko, P. A., Tkachuk, V. P. (1993). The Antenna Synthesis According to Amplitude Radiation Pattern: Numerical Methods and Algorithms, (in Russian). Kiev, Nauk. Dumka.

[5] Andriychuk, M. I. (1998). The Analytical-numerical Solution Method of the Nonlinear Problems of the Antenna Phase Synthesis. Proc. of IIIrd Intern. Seminar/Workshop on Direct and Inverse Problems of Electromagnetic and Acoustic Wave Theory (DIPED-98), Tbilisi, Georgia, pp. 86-89.

[6] Andriychuk, M. I., Zamorska, O. F. (2004). The Antenna Systems Synthesis According to the Amplitude Characteristics under Condition of Electromagnetic Compatibility. 2004 Second Intern. Workshop on Ultrawideband and Ultrashort Impulse Signals, Sevastopol, Ukraine. Sept. 2004, pp. 135-137.

[7] Andriychuk, M. I., Klakovych, L. M., Savenko, P. O., Tkach, M. D. (2005). Numerical Solution of Nonlinear Synthesis Problems of Antenna Arrays with Regard for Coupling of Radiators. Proc. of Vth International Conference on Antenna Theory and Techniques, 24-27 May 2005, Kyiv, Ukraine, pp. 213-216.

[8] Andriychuk, M., Zamorska, O. (2006). Waveguide Antenna Synthesis According to the Amplitude Radiation Characteristics in the Frequency Band. Proc. of $I^{\text {st }}$ European Conference on Antennas and Propagation. 6-10 November 2006. Nice, France, 6 p.

[9] Balanis, C. A. (2005). Antenna Theory: Analysis and Design. 3rd Edition. Hoboken, NJ, Wiley.

[10] Bityutskov, V. I. (2001). Bunyakovskii Inequality, in M. Hazewinkel, Encyclopedia of Mathematics. Springer.

[11] Bulatsyk, O. O., Katsenelenbaum, B. Z., Topolyuk, Y. P., and Voitovich, N. N. (2010). Phase Optimization Problems. Application in Wave Field Theory. Weinheim, Wiley-VCH.

[12] Cupradze, V. D. (1967). About Approximate Solution of Mathematical Physics Problem, (in Russian). Uspekhi Mat. Nauk, vol. 22, No 2, pp. 59-107. 
[13] Deuflhard, P. (2004). Newton Methods for Nonlinear Problems. Affine Invariance and Adaptive Algorithms. Series Computational Mathematics, 35, Springer.

[14] Dmitriev, V. I., Berezina, N. I. (1986). Numerical Methods of Solution of the Synthesis Problems for the Radiating Systems, (In Russian). Moscow, MGU.

[15] Feld, Ya. N. (1992). On the Quadratic Lemma in Electrodynamics. Sov. Phys. - Dokl., 37, pp. 235-236.

[16] Fourikis, N. (2000). Advanced Array Systems. Applications and RF Technologies. Phased Array Systems. Ascot park, Academic Press.

[17] Gelfand, I. M., Fomin, S. V. (2000). Calculus of Variations. Dover Publ.

[18] Kaklamani, D. I., Anastassiu, H. T. (2002). Aspects of the Method of Auxiliary Sources (MAS) in Computational Electromagnetics. IEEE Antennas \& Propagation Magazine, vol. 44, No 6, pp. 48-64.

[19] Katsenelenbaum, B., Marcader del Rio, L., Pereyaslavets, M., at al. (1998). Theory of Nonuniform Waveguides, London, IEE Series.

[20] Katsenelenbaum, B. Z. (2003). Electromagnetic Fields - Restriction and Approximation. Weinheim, Wiley-VCH.

[21] Katsenelenbaum, B. Z. (2006). High-Frequency Electrodynamics. Weinheim, Wiley-VCH.

[22] Khzmalyan, A. D., Kondratyev, A. S. (2003). The Phase-Only Shaping and Adaptive Nulling of an Amplitude Pattern. IEEE Trans. Antennas and Propag., vol. AP-51,No 2, pp. 264-272.

[23] Krasnoselsky, M. A., Zabreiko, P. P. (1975). Geometric Methods of Nonlinear Analysis, (in Russian). Moscow, Nauka.

[24] Kumar, A., Hristov, H. D. (1989). Microwave Cavity Antennas. Boston- London, Artech House.

[25] Landau, L., Lifshitz, E. (1984). Electrodynamics of continuous media. London, Pergamon Press.

[26] Markov, G. T., Petrov, B. M., Grudinskaya, G. P. (1979). Electrodynamics and Propagation of Waves, (in Russian). Moscow, Sov. Radio.

[27] Milligan, T. A. (2005). Modern Antenna Design. Hoboken, NJ, John Wiley \& Sons.

[28] Polak, E. (1971). Computational Methods in Application. A Unified Approach. New York, Academic Press.

[29] Savenko, P. O. (2002). Nonlinear Synthesis Problems of Radiating Systems. Lviv, Ukraine, IAPMM.

[30] Smirnov, V. I. (1958). Higher Mathematics, (in Russian). Vol. 4, Moscow, GIFML.

[31] Styeskal, H. 1, Herd, J. S. (1990). Mutual Coupling Compensation in Small Array Antennas. IEEE Trans. on AP, Vol. 38, No. 12, pp. 1971-1975.

[32] Tikhonov, A. N., Arsenin, V. Y. (1977). Solutions of Ill-Posed Problems. New York, Wiley.

[33] Vapnyarskii, I. B. (2001). Lagrange Multipliers, in M. Hazewinkel, Encyclopedia of Mathematics, Springer.

[34] Veinstein, L. A. (1998). The Electromagnetic Waves, (in Russian). Moscow, Radio i Svyaz.

[35] Vendik, O. G., Parnes, M. D. (2002). Antennas with Electrical Scanning, (in Russian). Saint Petersburg, Science Press.

[36] Vladimirov et al. (1985). Electromagnetic Compatibility of Radioelectronic Devices and Systems, (in Russian). Moscow, Radio i Svyaz.

[37] Voitovich, N. N., Katsenelenbaum, B. Z. Korshunova, E. N. , at al. (1989). Electrodynamics of Antennas with Semitransparent Surfaces, (in Russian). Moscow, Nauka.

[38] Weinberg, M. M., Trenogin, V. A. (1969). Theory of the Branching of Solutions of Nonlinear Equations, (in Russian). Moscow, Nauka. 